\title{
PrevGeo - A geoestatística aplicada em estudos de séries temporais: uma comparação com os métodos de Holt-Winters e Box e Jenkins
}

\author{
Sueli A. Mingoti, Ph.D. \\ Fernando Augusto Alves Glória, Bolsista de Iniciação Científica CNPq \\ Departamento de Estatística - Universidade Federal de Minas Gerais \\ e-mail: sueli@est.ufmg.br ; bodim@est.ufmg.br
}

\begin{abstract}
Neste artigo, apresentamos uma comparação de três metodologias utilizadas em séries temporais: a de Geoestatística, de Holt-Winters e Box-Jenkins. A comparação é feita utilizando-se duas séries reais extraídas do artigo de Pellegrini \& Fogliatto (2000) no qual os autores ajustam os modelos de Holt-Winters e o de Box-Jenkins.

Para obtenção das previsões via metodologia de Geoestatística, utilizou-se o programa computacional PrevGeo especialmente desenvolvido por Mingoti, Glória e Neves (2001). Este programa funciona junto ao software estatístico Minitab for Windowsß. e é muito fácil de ser utilizado.

As séries tratadas neste estudo correspondem, respectivamente, à demanda mensal de um produto fabricado por uma empresa do ramo de plásticos, localizada no Rio Grande do Sul, e à demanda mensal de uma determinada marca de refrigerantes (Montgomery et al., 1990).

O objetivo deste trabalho é mostrar que a metodologia de Geoestatística proposta em Mingoti e Neves (1999), é um método automático de fácil implementação, e que pode resultar em previsões satisfatórias.
\end{abstract}

Palavras-chave: Holt-Winters; Box-Jenkins; geoestatística; séries temporais.

A comparison among the Geostatistics, Holt-Winters and Box-Jenkins methodologies used for time series analysis is presented in this paper. Two time series, the monthly demand of a plastic product manufactured by a company located at Rio Grande do Sul and a monthly demand of some softdrink brand (Montgomery et.al 1990), are investigted. They were analysed by Pellegrini \& Fogliatto (2000) through Holt-Winters and Box-Jenkins methods. To generate the predictions by using Geostatistics methodology, a computer program named PrevGeo, was especially developed by Mingoti, Glória \& Neves (2001). This program works in the Minitab for Windows statistical software environment and it is very easy to use.

The main purpose of this paper is to show that Geostatistics as presented in Mingoti \& Neves (1999) can be considered as an automatic method for time series analysis being very simple to be used and producing satisfactory predictions.

Key-Words: Holt-Winters; Box-Jenkins; geostatistics; time series.

\section{Introdução}

Os métodos de previsão constituem ferramentas estatísticas importantes que podem auxiliar no processo de tomada de decisões. Numa empresa, por exemplo, calculando-se a previsão das vendas de um produto para os próximos meses, pode-se definir ações a serem tomadas na compra de matéria-prima para produção visando a minimização de prejuízos causados pela compra excedente de matéria-prima, ou perda financeira em função do não cumprimento de contratos previamente firmados com os clientes.

Dentre as várias metodologias existentes para tratamento de séries temporais destacam-se os modelos estocásticos de Box-Jenkins (1976), os modelos Bayesianos (Harrison e West,1987) e os modelos automáticos de Holt-Winters (Winters, 1960; Morettin \& Toloi, 1983) que são mais apropriados para séries de tamanhos pequenos.

Uma metodologia alternativa foi introduzida por Mingoti e Neves (1999) na qual os métodos de Geoestatística são utilizados para produzir modelos de projeção. Neste artigo, Mingoti \& Neves (1999) mostram através de um estudo de casos, que esta nova metodologia pode resultar em previsões de melhor qualidade que as de Box-Jenkins. Por ser um método de simples aplicação, e que não exige a identificação “à priori” de modelos estatísticos como 
é o caso da metodologia de Box e Jenkins, ela pode ser considerada como um método automático de predição constituindo-se numa alternativa ao método de Holt-Winters.

A proposta deste artigo é apresentar uma comparação da metodologia de Geoestatística para séries temporais com a de Holt-Winters e a de Box e Jenkins através de um estudo de casos.

Toda análise computacional foi produzida através de um programa computacional PrevGeo, especialmente desenvolvido por Mingoti, Glória \& Neves (2001). Este programa funciona no ambiente do software estatístico Minitab for Windows e estará disponível para download sem custo para o usuário no endereço www.est.ufmg.br.

As séries que serão tratadas neste artigo correspondem respectivamente, à demanda no período de outubro de 1993 a dezembro de 1997, de um produto de uma empresa de plásticos, localizada no Rio Grande do Sul e a série de dados reais de demanda de uma determinada marca de refrigerantes (Montgomery et al.,1990). As duas séries foram apresentadas em Pellegrini \& Fogliatto (2000) e analisadas via metodologias de Holt-Winters e Box$s^{\text {Jepkins. }} \underset{=}{=} \dot{\gamma}(t-s), \forall t \neq s \in T$,

A comparação dos modelos de projeção será feita através da análise do Erro Médio, Erro Absoluto Médio, Erro Quadrático Médio e Erro Absoluto Relativo Médio.

\section{Breve descrição das metodologias}

Nesta seção, faremos uma breve descrição das metodologias de previsão que serão comparadas neste artigo, dando maior enfoque à metodologia de Geoestatística por ser esta, menos conhecida.

\subsection{A metodologia de geoestatística em séries temporais}

Embora tenha sido inicialmente formulada para tratamento de dados geológicos (Matheron 1963), a metodologia de Geoestatística (Cressie, 1993) pode ser utilizada em outros contextos como na estimação de casos diagnosticados de AIDS nos municípios de Minas Gerais (Mingoti \& Pantuzzo, 1998; Mingoti, Rosa \& Leite, 2001), na estimação da taxa de mortalidade infantil (Cressie, 1993), ou na estimação da variabilidade de processos industriais (Mingoti \& Fidelis, 2001) entre outros. Basicamente a metodologia de Geoestatística pode ser aplicada quando se tem uma amostra aleatória de uma variável resposta contínua, na qual cada elemento amostral tem suas coordenadas de localização. A análise da variação da variável resposta em relação às coordenadas de localização da amostra possibilita a construção de um modelo estatístico usado para predizer-se o valor da variável resposta em localizações não previamente amostradas. O conhecimento do comportamento desta variação é obtido através de uma função chamada de semi-variograma e que é utilizada no procedimento de predição espacial chamado de krigagem (Matheron 1963; Krige,1951). Dado uma nova localização por exemplo, a krigagem permite que o valor da variável resposta para esta localização seja predito com base no comportamento dos valores amostrais da variável resposta em uma vizinhança de " $s$ ". Outras funções podem ser utilizadas para descrever a variabilidade espacial, entre elas destacam-se: o Madograma e Rodograma (Mingoti, 1996; Journel, 1988).

Em Mingoti \& Neves (1999), a metodologia de Geoestatística foi utilizada para fazer previsões da série de "número de manchas solares de Wolfer no período de 1770 a 1869", série bastante conhecida na área de séries temporais em virtude de sua alta variabilidade e dificuldade de predição. Os autores mostraram que, para esta série, a metodologia de Geoestatística resultava em melhores predições que o método de Box e Jenkins. Dentro do contexto de séries temporais o objetivo é estudar-se o comportamento da variável resposta $Z(t)$ que é medida no tempo $t$. Portanto, o tempo faz o papel da coordenada de localização da unidade amostral.

Para se proceder a análise dos dados temporais através dos métodos de Geoestatística é necessário a imposição de que o processo estocástico gerador dos dados amostrais seja intrinsicamente estacionário e isotrópico, ou seja, deve satisfazer as seguintes condições:

$$
E[Z(t)]=\mu, \forall t \in T ;
$$

onde $T$ é o conjunto de indexação de tempo. Estas condições implicam em dizer-se que: o processo tem média constante ao longo do tempo e para cada a variância da diferença é uma função que depende apenas da distância no tempo entre as observações $Z(t)$ e $Z(s)$, isto é, $|\mathrm{t}-\mathrm{s}|$, sendo $\gamma(\bullet)$ o semi-variograma do processo estocástico gerador da série e o variograma.

Alguns exemplos mais comuns de modelos de semivariograma são o modelo linear, o esférico, o senoide e o exponencial (Cressie, 1993). Na prática, o variograma é estimado a partir dos dados amostrais, 
sendo o estimador clássico não viciado usual de Matheron (1963), para o caso de séries temporais, definido por:

$$
2 \hat{\gamma}(h)=\frac{\sum_{t=1}^{n-h}[Z(t)-Z(t-h)]^{2}}{n-h}
$$

onde $h \in T=\{1,2, \ldots, n-1\}$,e $(n-h)$ é o número de pares , que estão a uma distância de $h$ unidades no período de amostragem. A função em (2.2) é chamada de variograma experimental. Para obter-se o semi-variograma experimental basta dividir-se por 2 o resultado da equação (2.2).

A previsão da variável resposta $Z($.) para um tempo " $s$ " qualquer, não pertencente à série observada, é obtida utilizando-se a técnica de predição espacial denominada Kriging Ordinário (Cressie, 1993, 1990). Neste caso, o preditor linear não viciado de $Z(s)$ é dado por:

$$
\hat{Z}(s)=\sum_{i=1}^{n} \lambda_{i} Z\left(t_{i}\right) \quad s>t_{n}
$$

onde

$$
\text { , e } Z\left(t_{1}\right), Z\left(t_{2}\right), \ldots, Z\left(t_{n}\right) \text { repre- }
$$

sentam respectivamente os valores observados da série ou de uma vizinhança usada na previsão de $Z(s)$. Os coeficientes $\lambda_{i}$ são obtidos através da seguinte equação:

$$
\lambda_{0}=\Gamma_{0}^{-1} \gamma_{0}
$$

onde,

$$
\gamma_{0}=\left(\gamma\left(s-t_{1}\right), \gamma\left(s-t_{2}\right), \ldots, \gamma\left(s-t_{n}\right), 1\right)^{\prime}
$$

e $\Gamma_{0}$ é a matriz $(n+1) x(n+1)$ dada por:

$$
\Gamma_{0}=\left\{\begin{array}{cl}
\gamma\left(t_{i}-t_{j}\right), i=1, \ldots, n, j=1, \ldots, n \\
1 & , i=n+1, j=1, \ldots, n \\
0 & , i=n+1, j=n+1 \\
1 & , i=1, \ldots, n, j=n+1
\end{array}\right.
$$

sendo que $\gamma(\bullet)$ representa o semi-variograma do processo e $\lambda_{0}$ é o vetor contendo os coeficientes $\lambda_{i}, i=1,2, \ldots, n$. O estimador linear $\hat{Z}(s)$ assim construído, é o que minimiza a variância do erro de predição definido por:

e cuja variância é dada por:

$$
\hat{\operatorname{Var}}(\operatorname{erro}(s))=\hat{\sigma}^{2}(s)=\lambda_{0}^{\prime} \gamma_{0}
$$

Um intervalo de $(1-\alpha)(100 \%)$ de confiança para o verdadeiro valor de $Z(s)$ pode ser obtido utilizando-se a distribuição normal isto é: $\hat{Z}(s) \pm z_{\alpha} / 2 \hat{\sigma}(s)$. É importante destacar que a variância do erro de predição permanece constante na previsão de valores cujas coordenadas mantém a mesma relação de distância com a vizinhança usada para predição, ou seja, se estamos interessados em prever os valores de $Z\left(s_{01}\right)$ e $Z\left(s_{02}\right)$, , e se a distância de aos seus vizinhos de predição for igual à distância de em relação aos seus vizinhos, a variância do erro associado à previsão de $Z($ ) será igual a variância do erro associado à previsão de $Z(\quad$ ), considerando-se que as duas vizinhanças contém o mesmo número de vizinhos. Em séries temporais isto significa dizer que, se estamos interessados em fazer previsão a $h$ passos à frente à partir de uma origem fixa, considerando-se sempre o mesmo número de vizinhos , as previsões que serão geradas terão todas a mesma variância do erro de predição ao contrário do que ocorre na metodologia de Box e Jenkins por exemplo, na qual a variância do erro aumenta à medida que se vai distanciando da origem de previsão.

A metodologia padrão de Geoestatística requer que os valores de , necessários para a implementação das equações (2.4) a (2.8), sejam obtidos através da identificação de um modelo de variograma teórico do processo, o que é feito a partir da função de variograma experimental dada em (2.2) e do ajuste dos parâmetros do modelo identificado. Entretanto, no caso de séries temporais esta fase pode ser eliminada se considerarmos que os valores amostrais obtidos através de (2.2) são próximos dos valores teóricos do variograma do processo (Mingoti \& Neves, 1999) e se os utilizarmos os valores do semi-variograma experimental nas equações (2.4) a (2.8). Isto entretanto, deve ser feito com cautela uma vez que o variograma amostral como definido em (2.2) não tem a propriedade de ser condicionalmente não negativo definido (c.n.d), propriedade esta requerida para que uma função matemática possa representar o variograma teórico de um processo estocástico (Cressie, 1993). O fato do variograma experimental não ser "c.n.d." faz com que eventualmente, dependendo da vizinhança escolhida para se efetuar a 
predição em uma localização " $s^{\prime \prime}$ qualquer, tenhamos situações nas quais o erro de predição tem uma variância negativa, algo inconsistente com a definição de variância. No entanto, na prática este problema pode ser contornado se escolhermos a vizinhança de predição de modo a evitar-se aquelas que geram este tipo de inconsistência.

É interessante observar que a equação de predição em (2.3) se assemelha a de um processo Auto Regressivo.

A imposição de que , permite que o processo seja não estacionário dentro da classe de Box e Jenkins, ou seja, seja um modelo "não estacionário de fronteira" que em geral, pode ser corrigido através de diferenciação de ordem 1 .

\subsection{O modelo de suavização exponencial de Holt-Winters}

\subsubsection{Modelo sazonal multiplicativo}

O modelo sazonal multiplicativo de Holt-Winters (Pellegrini \& Fogliatto, 2000) é utilizado na modelagem de dados sazonais onde a amplitude de um ciclo sazonal cresce em função do tempo ou seja, a diferença entre o maior e o menor ponto de demanda nos ciclos cresce com o passar do tempo. De acordo com a notação apresentada por Pellegrini \& Fogliatto (2000), o modelo multiplicativo de Holt-Winters para a série $Z(t)$ é definido por:

$$
Z_{t}=\left(b_{1}+b_{2} t\right) c_{t}+e_{t}
$$

onde $b_{1}$ é o componente permanente de média, $b_{2}$ é um componente de tendência linear, $c_{t}$ é um fator sazonal multiplicativo e $e_{t}$ representa o erro aleatório, independente e identicamente distribuído.

A utilização deste modelo requer o prévio conhecimento da duração do ciclo sazonal existente na série temporal, relativamente à unidade de tempo em que os dados são coletados. Assim se uma série apresentar sazonalidade anual com dados coletados mensalmente, seu ciclo sazonal será $L=12$ meses. A relação existente entre os fatores sazonais e a duração do ciclo é . Caso a série não apresente tendência o componente $b_{2}$ será igual a zero.

\subsubsection{Modelo sazonal aditivo}

O modelo aditivo de Holt-Winters é utilizado na modelagem de dados sazonais onde a amplitude de ciclo sazonal permanece constante com o passar do tempo. De acordo com a notação de Pellegrini \& Fogliatto (2000), o modelo aditivo de Holt-Winters para a série $Z(t)$ é definido por:

$$
Z_{t}=b_{1}+b_{2} t+c_{t}+e_{t}
$$

onde $b_{1}$ é o componente permanente de média, $b_{2}$ é um componente de tendência linear, $c_{t}$ é um fator sazonal aditivo e $e_{t}$ representa o erro aleatório, independente e identicamente distribuído, sendo que para séries que não apresentam tendência o componente $b_{2}$ é igual a zero. Ao contrário do modelo multiplicativo, o modelo sazonal aditivo é adequado no estudo de séries temporais onde a amplitude do ciclo sazonal independe do nível médio da série. Neste caso, a soma dos fatores sazonais é igual a zero, isto é

$$
\sum_{t=1}^{L} c_{t}=0 \text {. }
$$

\subsection{Modelos de Box-Jenkins}

Os modelos de Box-Jenkins exploram a estrutura de autocorrelação do processo estocástico gerador dos dados da série temporal. Através das funções de autocorrelação e autocorrelação parciais, identifica-se um modelo estatístico dentro da classe de processos estocásticos ARMA para descrever a série e seus parâmetros são estimados (Box e Jenkins, 1976). Quando o modelo passa pela fase de verificação sem problemas, este pode ser utilizado para gerar previsões de valores futuros da série.

De um modo geral os modelos ARMA $(p, q)$ estacionários podem ser descritos da seguinte forma:

$$
\begin{aligned}
& Z_{t}=\delta+\phi_{1} Z_{t-1}+\phi_{2} Z_{t-2}+\ldots \\
& +\phi_{p} Z_{t-p}-\theta_{1} a_{t-1}-\theta_{2} a_{t-2}-\ldots \\
& -\theta_{q} a_{t-q}+a_{t}
\end{aligned}
$$

onde $Z(t)$ é a característica de interesse medida na amostra $t$ (ou unidade amostral $t$ ), $a_{t}$ é o erro aleatório com distribuição normal de média zero e variância igual a $\sigma_{a}^{2}$, e $\left(\phi_{1}, \phi_{2}, \ldots, \phi_{p}, \theta_{1}, \theta_{2}, \ldots, \theta_{q}, \delta, \sigma_{a}^{2}\right)$ são os parâmetros do modelo. Estes parâmetros devem satisfazer certas condições de estacionariedade e invertibilidade do processo. Alguns dos modelos mais simples dentro desta classe são: o Auto-Regressivo e o Média Móvel. Para maiores detalhes técnicos sobre os modelos de Box e Jenkins sugerimos a leitura de Brockwell \& Davies (1991). 


\section{Implementação da metodologia de meoestatística: apresentação do programa computacional PrevGeo}

Nesta seção apresentamos o programa PrevGeo elaborado por Mingoti, Glória e Neves (2001) para a implementação da metodologia de Geoestatística em análise de séries temporais. Para exemplificação será utilizada a série 1 que descreve a demanda mensal de uma fábrica de plásticos do Estado do Rio Grande do Sul, no período compreendido entre outubro de 1993 a dezembro de 1997 ( Pellegrine \& Fogliatto, 2000). Esta série tem um total de 51 observações ( Gráfico 1). Vamos fixar como origem da previsão o ponto $t=45$ com o objetivo de obter previsões para os 6 valores futuros da série a partir desta origem, isto é vamos gerar previsões para 6 passos à frente. A vizinhança escolhida para exemplificação é igual a 2, ou seja a previsão de $Z(s)$ será obtida à partir dos 2 valores observados (ou preditos) de $Z(t)$ que antecedem a $Z(s)$.

Para obtermos previsões usando o programa PrevGeo, basta chamarmos a macro dentro do sistema Minitab for Windows fornecendo as seguintes informações: a coluna que contém os dados observados da série, o número de previsões a serem feitas, a vizinhança de predição a ser utilizada e o nível de confiança que será usado no cálculo dos intervalos de confiança para os valores verdadeiros da série (Figura 1). A partir destas informações o programa passa a executar os comandos necessários para o cálculo do variograma experimental (equação 1.1) e para implementação da equação de previsão (2.3). Como saída o programa mostra as previsões a um passo, as previsões pontuais e por intervalo geradas para os valores futuros da série a partir da origem de predição escolhida e os valores dos coeficientes de projeção

. Além disso, são impressas medidas que permitem ao usuário analisar a qualidade das predições geradas, isto é, o Erro Médio (EM), Erro Absoluto Médio (ABSERROM), Erro Quadrático Médio (EQM) com sua raiz quadrada (SQRTEQM), e o Erro Absoluto Relativo Médio (ABSERREM) considerando-se as previsões feitas a partir da origem fixa, assim como o Erro Médio $(E M 1 P)$ e o Erro Quadrático Médio $(E Q M 1 P)$ com sua respectiva raiz quadrada (SQRTEQM1) considerando-se as previsões à um passo a frente, as quais no programa são chamadas de Série Suavizada. O usuário poderá guardar os coeficientes de projeção e as previsões com os respectivos intervalos de confiança. Poderá ainda, fazer um gráfico no qual a série real com as projeções e intervalos de confiança são plotados. A Figura 2 mostra parte da execução do programa PrevGeo e os resultados obtidos para a série 1 . Pelas projeções obtidas fica claro que o modelo de Geoestatística com vizinhança 2 não é adequado para se fazer projeções para esta série. Os intervalos de confiança tem limites inferiores negativos, algo incompatível com a natureza da série analisada. As variâncias dos erros de predição são muito elevadas.
Gráfico 1 - Apresentação das séries analisadas

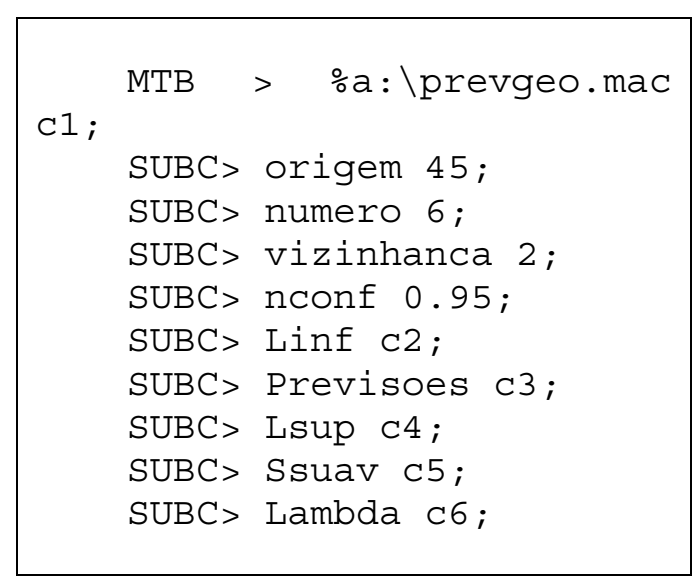

Figura 1 - Comandos para iniciar a execução do programa PrevGeo 
Mingoti, Sueli A. ; Glória, Fernando A. A. ; - PrevGeo - A geoestatística aplicada em estudos ...

Séries, Previsões, Limites e Serie Suavizada

$\begin{array}{crcccc}\text { Row } & \text { C1 } & \text { Linferior } & \text { Prev } & \text { Lsuperior } & \text { Ssuavizada } \\ 46 & 58 & -694.761 & 52.4466 & 799.654 & 52.45 \\ 47 & 90 & -692.662 & 54.5449 & 801.752 & 61.48 \\ 48 & 78 & -692.141 & 55.0662 & 802.273 & 97.95 \\ 49 & 248 & -692.011 & 55.1957 & 802.403 & 75.02 \\ 50 & 686 & -691.979 & 55.2279 & 802.435 & 290.23 \\ 51 & 1370 & -691.971 & 55.2359 & 802.443 & 794.81\end{array}$

Erros de Previsão

\section{Data Display}

$\begin{array}{lr}\text { EM } & -367.047 \\ \text { EQM } & 360911 \\ \text { SQRTEQM } & 600.759 \\ \text { EM1P } & -11.5793 \\ \text { EQM1P } & 145013 \\ \text { SQTEQM1P } & 380.806 \\ \text { ABSERROM } & 367.047 \\ \text { ABSERREM } & 0.573388\end{array}$

Figura 2 - Previsões geradas para a série 1 pela metodologia de Geoestatística - vizinhança 2 -

Para a série 1 os coeficientes de projeção são: Deste modo, as previsões para $Z(s), s=46,47,48,49,50,51$, com origem de previsão em $t=45$, são geradas a partir da equação:

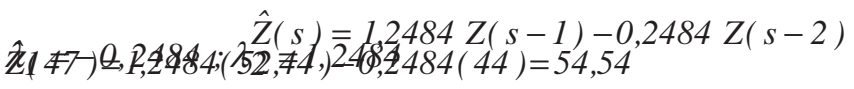

Assim por exemplo, a previsão para $Z(46)$ é dada por:

$$
\begin{aligned}
\hat{Z}(46) & =1,2484 Z(45)-0,2484 Z(44)= \\
& =1,2484(44)-0,2484(10)=52,44
\end{aligned}
$$

enquanto que a previsão para $Z(47)$ é dada por:

assim sucessivamente. $\mathrm{O}$ desvio padrão do erro de previsão a 6 passos à frente neste caso, é estimado por 381,23 para $Z(s), s=46,47,48,49,50,51$.

Como exemplo da série suavizada, a previsão à um passo a frente para Z(48) é obtida pela equação:

$$
\begin{aligned}
\hat{Z}(48) & =1,2484 Z(47)-0,2484 Z(46)= \\
& =1,2484(90)-0,2484(58)=97,95
\end{aligned}
$$

O usuário poderá executar o programa para vários valores de vizinhanças diferentes escolhendo no final aquela que gera as melhores previsões. Embora, teoricamente a vizinhança possa ser escolhida no conjunto $\{2,3, \ldots, s\}$, modelos com vizinhança muito grande devem ser evitados em virtude da baixa qualidade de estimação do variograma experimental e dos coeficientes que fazem parte das equações de previsão.

Para a série 1, os 3 melhores modelos consistentes com a condição "c.n.d" estão apresentados na Tabela 1. Como pode ser observado, a vizinhança igual a 25 foi a que forneceu o melhor ajuste em termos de $E M \mathrm{e}$ $E Q M$. No entanto, se considerarmos as previsões a um passo geradas pelo modelo e o erro absoluto relativo médio, veremos que a melhor vizinhança é a de ordem 17.

O Gráfico 2 mostra uma comparação dos valores reais da série com os valores previstos considerando-se as 3 vizinhanças, enquanto que o Gráfico 3 contém os valores reais da série, os valores previstos usando a vizinhança igual a 25 , os limites superior e inferior do intervalo de $95 \%$ de confiança para os verdadeiros valores da série juntamente com os valores para a série suavizada.

Tabela 1 - Melhores ajustes para a série 1, em relação ao Erro Quadrático Médio (EQM)

\begin{tabular}{lrrr}
\hline \multicolumn{1}{c}{ Vizinhança } & \multicolumn{1}{c}{16} & 17 & \multicolumn{1}{c}{25} \\
\hline EM & $-145,892$ & $-170,708$ & 10,8972 \\
EQM & 28885,5 & 36663,3 & 16093,8 \\
SQRTEQM & 169,9573 & 191,4766 & 126,8613 \\
EM1P & 38,4511 & 29,1652 & 91,7189 \\
EQM1P & 56051,3 & 55004,5 & 146901 \\
SQRTEQM1P & 236,7516 & 234,5304 & 383,2767 \\
ABSERROM & 156,701 & 173,193 & 121,764 \\
ABSERREM & 1,45077 & 1,68222 & 1,00592 \\
\hline
\end{tabular}




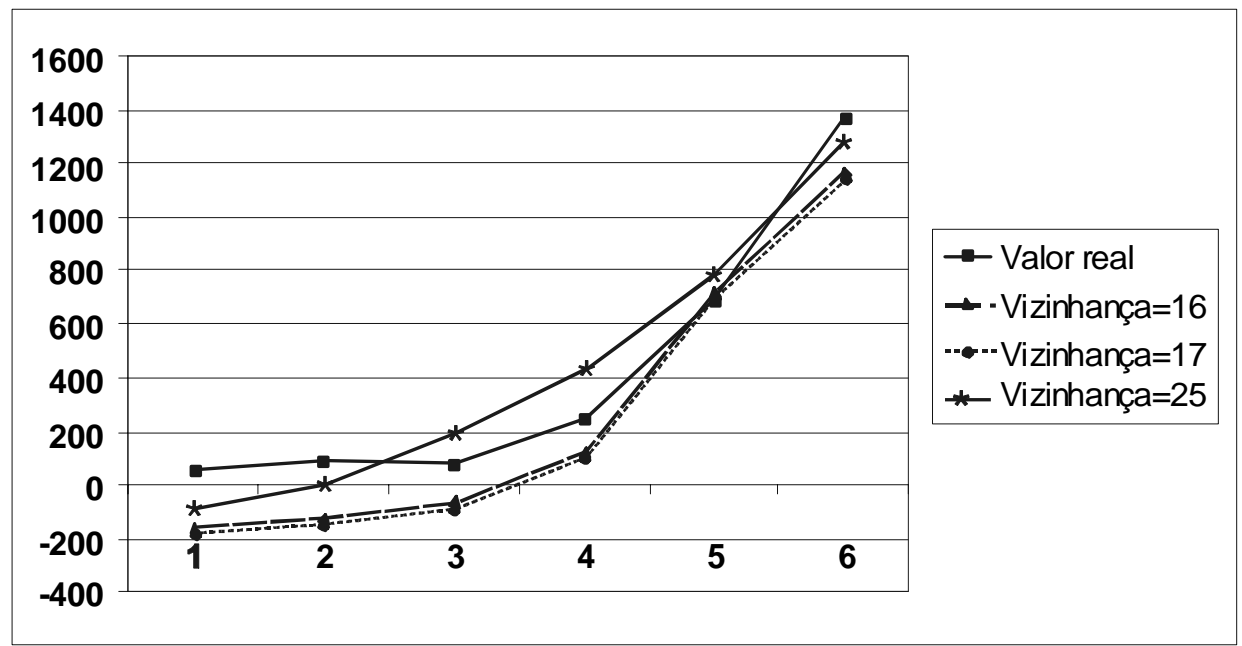

Gráfico 2 - Comparação entre os valores reais da série 1 e as previsões geradas pelos modelos de Geoestatística - vizinhanças 16,17,25

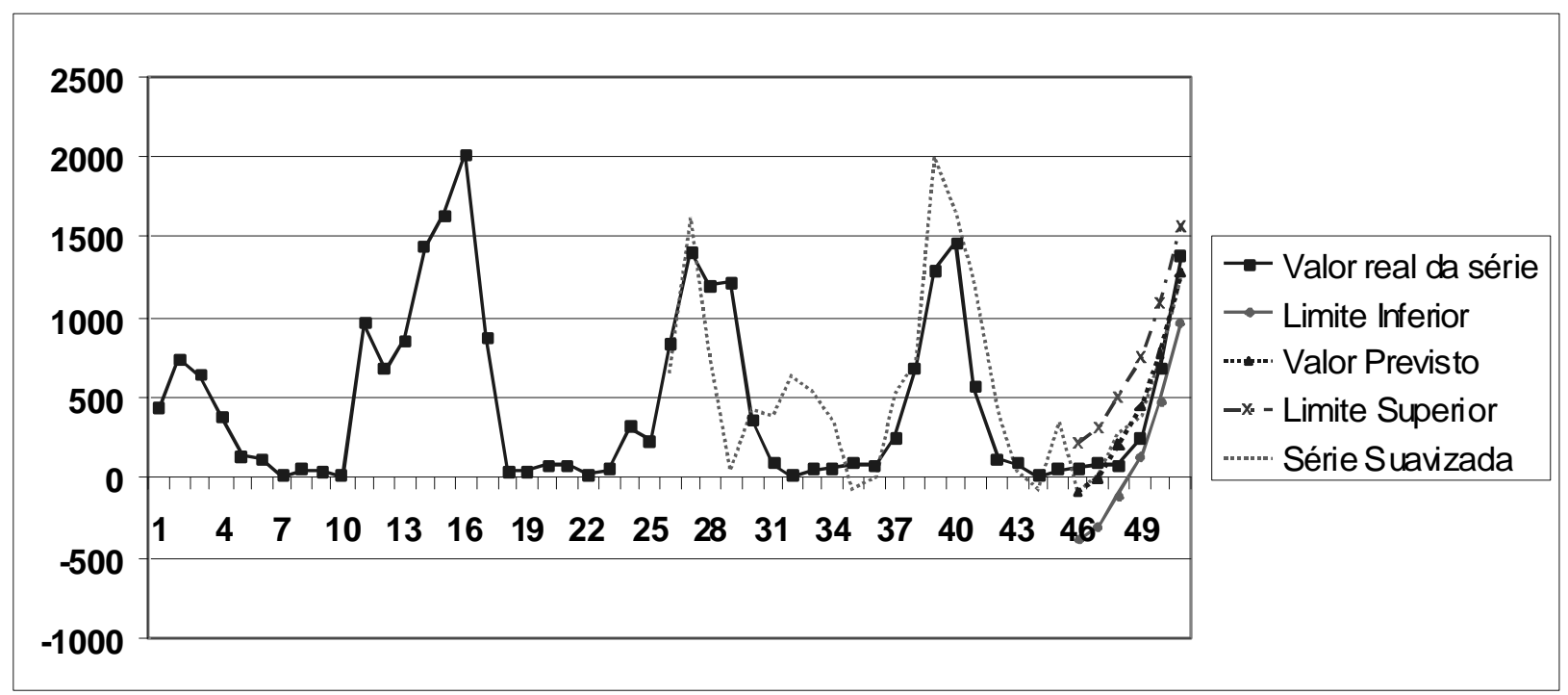

Gráfico 3 - Comparação entre os valores reais da série 1 e os valores previstos - vizinhança igual a 25

\section{Estudo de casos: comparação da cetodologia de ceoestatística com as cetodologias de Holt-Winters e Box- Jenkins}

Pellegrine \& Fogliatto (2000) analisaram as duas séries apresentadas no Gráfico 1 através das metodologias de Holt-Winters e Box-Jenkins. Os valores dos Erros Quadráticos Médios a um passo para estas duas séries, nas duas metodologias foram apresentados em Pellegrini \& Fogliatto (2000). Para a série 1 o modelo de Box-Jenkins escolhido pelos autores para representar o comportamento da série foi um $\operatorname{ARMA}(14,13)$, ou seja um modelo constituído de 27 parâmetros. O modelo sazonal aditivo de Holt-Winters foi, segundo os autores, o mais apropriado para representar a série 1 dentro dessa classe de modelos. Os valores de
EQM a um passo apresentados por Pellegrini \& Fogliatto (2000) foram respectivamente iguais a: $E Q M_{B J}=53751 E{ } M_{H W}=87642$. Os três melhores modelos consistentes com a condição “c.n.d." para a série 1, considerando-se o valor de EQM $1 P$ e utilizando-se a metodologia de

Geoestatística, estão mostrados na Tabela 2. Pode ser notado que o modelo com vizinhança igual a 15 foi o melhor $\left(E Q M_{G}=48756,1\right)$ sendo que os três melhores modelos produzidos pela metodologia de Geoestatística forneceram Erros Quadráticos Médios a um passo menor que os modelos de Box e Jenkins e Holt-Winters apresentados em Pellegrini \& Fogliatto (2000). Além disso, a equação de predição dos modelos de Geoestatística têm um número de parâmetros bastante inferior ao do modelo de Box e Jenkins ajustado por estes autores. 
Tabela 2 - Melhores ajustes para a série 1 em relação ao Erro Quadrático Médio a Um Passo (EQM1P)

\begin{tabular}{lrrr}
\hline Vizinhança & \multicolumn{1}{c}{14} & \multicolumn{1}{c}{15} & \multicolumn{1}{c}{17} \\
\hline EM & $-255,712$ & $-237,753$ & $-170,708$ \\
EQM & 70277,5 & 61292,2 & 36663,3 \\
SQRTEQM & 265,099 & 247,5726 & 191,4766 \\
EM1P & 15,971 & 25,57 & 29,1652 \\
EQM1P & 52004,5 & 48756,1 & 55004,5 \\
SQRTEQM1P & 228,045 & 220,8078 & 234,5304 \\
ABSERROM & 255,712 & 237,753 & 173,193 \\
ABSERREM & 2,18390 & 2,04234 & 1,6222 \\
\hline
\end{tabular}

Para a série 2, os melhores modelos apresentados por Pellegrini \& Fogliatto (2000) foram o $A R M A(2,1)$ de Box e Jenkins e um modelo sazonal multiplicativo de Holt-Winters. Os valores dos Erros Quadráticos Médios a um passo fornecidos pelos autores para este modelos foram respectivamente, iguais a:

$E Q M_{B J}=22733, E Q M_{H W}=6909$. A Tabela 3 mostra os quatro melhores modelos consistentes com a condição "c.n.d" para a série 2 obtidos pela metodologia de Geoestatística levando-se em consideração como medida de qualidade de ajuste os menores valores para o EQMIP. Como 38 e 40 são valores de vizinhanças muito elevados, a preferência foi dada ao modelo com vizinhança igual a 11. Este modelo apresenta um Erro Quadrático Médio a um passo menor que o de Box e Jenkins mas maior que o de Holt-Winters, o mesmo acontecendo para o modelo com vizinhança igual a 10. Para a série 2 a metodologia de Geoestatística não conseguiu produzir um modelo alternativo com um número de parâmetros menor que o de Box e Jenkins.

Tabela 3 - Melhores ajustes para a série 2 em relação ao Erro Quadrático Médio a Um Passo ( EQM1P)

\begin{tabular}{|l|c|c|c|c|c|c|}
\cline { 2 - 7 } \multicolumn{1}{c|}{} & CAI & TYO & ORD & SFO & LGW & YMX \\
\hline Cairo (CAI) & 1 & $1 / 3$ & 8 & 3 & 3 & 7 \\
\hline Tóquio (TYO) & 3 & 1 & 9 & 3 & 3 & 9 \\
\hline Chicago (ORD) & $1 / 8$ & $1 / 9$ & 1 & $1 / 6$ & $1 / 5$ & 2 \\
\hline San Francisco (SFO) & $1 / 3$ & $1 / 3$ & 6 & 1 & $1 / 3$ & 6 \\
\hline Londres (LGW) & $1 / 3$ & $1 / 3$ & 5 & 3 & 1 & 6 \\
\hline Montreal (YMX) & $1 / 7$ & $1 / 9$ & $1 / 2$ & $1 / 6$ & $1 / 6$ & 1 \\
\hline
\end{tabular}

\section{Considerações finais}

A proposta deste artigo foi mostrar a metodologia de Geoestatística proposta em Mingoti e Neves (1999) para a análise de séries temporais e o programa computacional PrevGeo desenvolvio por Mingoti, Glória e Neves (2001) para sua implementação. Foi feita uma comparação desta metodologia com as de Holt-Winters e de Box-Jenkins através de um estudo de casos considerando-se as séries analisadas em Pellegrini \& Fogliatto (2000). Pelos resultados observados vimos que a metodologia de Geoestatística apresentou resultados satisfatórios, sendo que para a série 1 os valores dos Erros Quadráticos Médios obtidos para os modelos de Geoestatística foram menores que aqueles fornecidos nas outras duas metodologias. Já para a série 2, os resultados apesar de não terem sido melhores que aqueles obtidos pela metodologia de Holt-Winters, foram bastante satisfatórios e melhores que os obtidos na metodologia de Box-Jenkins. Em vista do fato de que, a aplicação da metodologia de Geoestatística para ajuste de séries temporais não exige do usuário comum nenhum reconhecimento específico de modelo estatístico para se obter as previsões, esta pode ser considerada como uma metodologia alternativa interessante. No entanto, é importante que o usuário tenha como hábito fazer uma análise das previsões resultantes, de modo a escolher a melhor vizinhança para gerar as previsões mantendo como critérios, a parcimônia e a qualidade de ajuste do variograma experimental, peça fundamental na determinação dos coeficientes que fazem a ponderação de valores na equação de predição.

\section{Referências bibliográficas}

BOX,G.P., JENKINS,G.M. (1976) Time series analysis: forecasting and control.New York:Holden Day.

BROCKWELL, P. J., DAVIES, R. (1991) Time series: theory and methods. New York: Springer Verlag.

CRESSIE, N. (1993) Statistics for espatial data. New York: John Wiley \& Sons.

CRESSIE, N. (1990) The origins of kriging. Mathematical Geology, 22, 239-252.

HARRISON, P.J., WEST, M. (1987) Practical bayesian forecasting. The Statistician, 36, 115-125.

JOURNEL,A.G.(1988) New distance measures:the route forward truly non-gaussian geostatistics.Mathematical Geology,20,4, 459-475.

KRIGE, D. G. (1951) A statistical approach to some basic mine valuation problems on the Witwatersrand. Journal of Chemical, Metallurgical and Mining Society of South Africa, 52, 119-139.

MATHERON, G. (1963) Principles of geostatistics. Economic Geololy, 58,1246-1266. 
MINGOTI, S.A., FIDELIS, M.T. (2001) Aplicando a geoestatística no controle estatístico de processos. Revista Produto \& Produção, 5,2,55-70.

Mingoti, S. A., GLÓRIA, F. A. A., NEVES, O. F. (2001) Aplicando a geoestatística na previsão de análise de séries temporais. Caderno de Resumos da X Semana de Iniciação Científica da UFMG, Belo Horizonte, 162.

MINGOTI, S.A.,ROSA, G., LEITE, A.G. (2001) Aplicando a geoestatística na descrição do número de casos diagnosticados de AIDS nos municípios de Minas Gerais no período de 1996 a 1999. Anais da 46a. Reunião Annual da RBRAS, 9a. SEAGRO, ESALQ/Piracicaba, 222-225.

MINGOTI, S. A., NEVES, O. F. (1999) A metodologia de geoestatística como alternativa na análise de séries temporais. Revista Escola de Minas (REM), 52,3, 182-187.

MINGOTI, S. A., PANTUZZO, A. (1998) E. Predição do número total de casos diagnosticados de AIDS dos municípios de Minas Gerais através de técnicas de estatística espacial. Revista de Matemática e Estatística da UNESP, 16, 59-80.

MINGOTI, S. A. (1996) As funções de madograma e rodograma como alternativas para descrever a variabilidade espacial dos dados. Revista Escola de Minas ( REM ), 50, 2, 71-74.

MONTGOMERY, D.C.; JOHNSON, L.A.;

GARDINER, J.S (1990) Forecasting and time series analysis, 2a. ed. New York: McGraw-Hill, Inc.

MORETTIN, P.A., TOLOI, C.M.C. (1981) Modelos para previsão em séries temporais. Vol. 1, 130.

Colóquio Brasileiro de Matemática, Poços de Caldas.

PELLEGRINI, F. R., FOGLIATTO, F. S. (2000)

Estudo comparativo entre os modelos de HoltWinters e de Box-Jenkins para previsão de demanda sazonal. Revista Produto \& Produção, 4, 2,72-85.

WINTERS, P. R. (1960) Forecasting sales by exponentially weighted moving averages.

Management Science, 6,3,324-342.

\section{Agradecimentos}

Nossos agradecimentos ao CNPq pelo apoio financeiro que possibilitou a execução deste trabalho. 